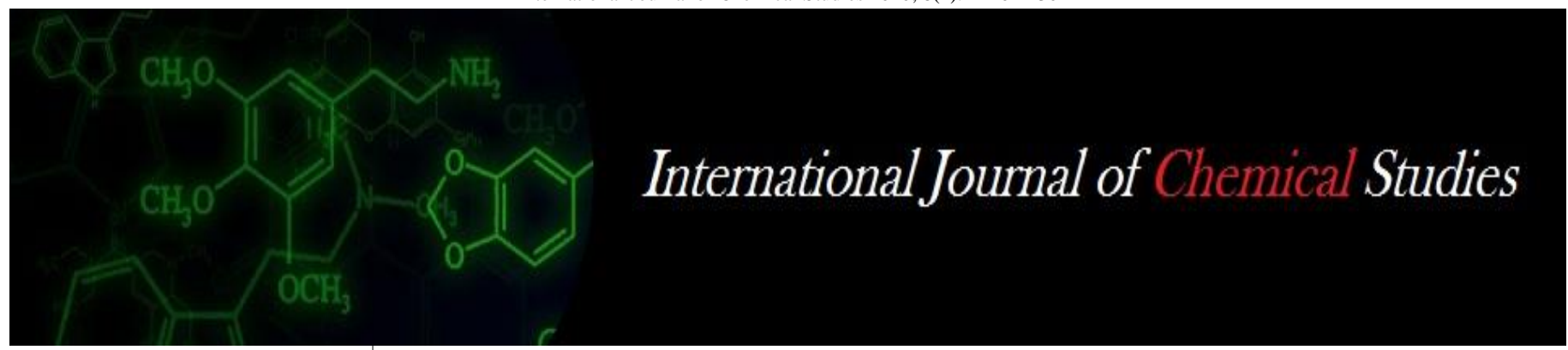

P-ISSN: 2349-8528

E-ISSN: 2321-4902

IJCS 2020; 8(1): 2126-2130

(C) 2020 IJCS

Received: 16-11-2019

Accepted: 18-12-2019

\section{Rehana Jan}

Sher-e-Kashmir University of Agricultural Sciences \& Technology of Kashmir, Shalimar, Srinagar, Jammu and Kashmir India

\section{JA Wani}

Sher-e-Kashmir University of Agricultural Sciences \& Technology of Kashmir, Shalimar, Srinagar, Jammu and Kashmir India

\section{MA Dar}

Sher-e-Kashmir University of Agricultural Sciences \&

Technology of Kashmir,

Shalimar, Srinagar, Jammu and Kashmir India

\section{WM Wani}

Sher-e-Kashmir University of

Agricultural Sciences \&

Technology of Kashmir,

Shalimar, Srinagar, Jammu and Kashmir India

\section{SA Mir}

Sher-e-Kashmir University of Agricultural Sciences \& Technology of Kashmir, Shalimar, Srinagar, Jammu and Kashmir India

\section{JA Khan}

Sher-e-Kashmir University of Agricultural Sciences \& Technology of Kashmir, Shalimar, Srinagar, Jammu and Kashmir India

Corresponding Author: Rehana Jan Sher-e-Kashmir University of Agricultural Sciences \& Technology of Kashmir, Shalimar, Srinagar, Jammu and Kashmir India

\section{Assessing fruit quality of high density apple cv. red velox by application of different levels of $N, P$ and $K$ under temperate conditions of Kashmir valley}

\author{
Rehana Jan, JA Wani, MA Dar, WM Wani, SA Mir and JA Khan
}

DOI: https://doi.org/10.22271/chemi.2020.v8.i1af.8583

\begin{abstract}
The present study was conducted at Sher-e-Kashmir University of Agricultural Sciences and Technology of Kashmir, Shalimar Campus Srinagar at High Density Apple Block of Division of Fruit Science for two consecutive years 2017-2018 and 2018-2019 with an objective to observe the influence of different levels and combinations of nitrogen, phosphorous and potassium on quality parameters of High Density Apple cv. Red velox. The results of the study revealed that the apple fruit quality viz., SSC, sugars, reducing and non-reducing sugars were significantly influenced with highest levels of phosphorus and potassium (i.e. $\mathrm{P}_{3}$ and $\mathrm{K}_{3}$ ) coupled with $\mathrm{N}_{2}$ level of nitrogen during 2017 and 2018 respectively when nutrients were applied in combination. Maximum soluble solids content (14.31, 15.04; 14.26, 14.87 and 14.23, 14.82 ${ }^{0}$ Brix $)$, total sugars $(9.685,9.897 ; 9.444,9.646$ and $9.472,9.687 \%)$, reducing sugars $(6.937,7.015 ; 6.985$, 7.047 and $7.005,7.067 \%)$, non-reducing sugars $(2.492,2.632 ; 2.366,2.479$ and $2.371,2.473 \%$ ) was obtained in $\mathrm{N}_{2}$ level of nitrogen coupled with highest level of $\mathrm{P}$ and $\mathrm{K}$ i.e., $\mathrm{P}_{3}$ and $\mathrm{K}_{3}$. Among the interaction effects, the treatment combination of $\mathrm{N}_{2} \mathrm{P}_{3} \mathrm{~K}_{3}$ recorded maximum SSC (14.50, $\left.15.19{ }^{\circ} \mathrm{Brix}\right)$, total sugars $(9.810,10.05 \%)$, reducing sugars $(7.20,7.39 \%)$ and non-reducing sugars $(2.61,2.73 \%)$ resulted in significant increase in quality attributes of High Density Apple cv. Red velox.
\end{abstract}

Keywords: Apple, high density planting, nitrogen, phosphorus, potassium, and quality attributes

\section{Introduction}

Apple (Malus domestica Borkh) is an important member of family Rosaceae and is widely cultivated in temperate regions of the world. The state of Jammu and Kashmir is bestowed with a particularly well suited climate for production of temperate fruits like Pear, Peach, Plum, Almond particularly Apple. Apple is the principal fruit crop of Jammu and Kashmir, accounting for 48.14 per cent of area and 77.26 per cent of total fruit production. It occupies an area of 16, 2971hectares with a total production of 18, 51,723 Metric tonnes and productivity of $12.16 \mathrm{t} /$ ha (Anonymous, 2019) ${ }^{[1]}$.

Providing adequate nutrition is the outmost concern for enhancing the yield as well as quality of apple. High density planting is one of the advanced techniques that have proven significantly good in increasing not only the productivity per unit area but also in improving the fruit quality (Singh, 2005) ${ }^{[13]}$. It has been observed that fertilizer requirements in high density arrangements are greater when compared to conventional planting. The effect of closer planting on fruit quality is mainly marked by the changes in quantity and quality of intercepted light and the partitioning of assimilate between vegetative and reproductive shoots (Policarpo et al. 2006) ${ }^{[9]}$. With proper nutrient management, this phenomenon could be balanced and hence will be helpful in improvement of fruit quality. With the increase in the plant and fruit number per unit area in dense planting, the nutrient management becomes the first and most important consideration. Therefore, nutrient management is essential for optimum plant growth, development and for increasing the fruit quality (Singh and Singh, 2007) ${ }^{[15]}$.

Nitrogen, phosphorus and potassium are key elements required by the apple tree for various important functions like growth of tissues, formation of amino acids and proteins, chlorophyll development, water uptake, transpiration, manufacture and translocation of sugars and starches, and quality of fruit. The amount of nitrogen, phosphorus and potassium required by plants is of great significance in fruit cultivation. A small change in nitrogen, phosphorus and potassium content in tissue has a great effect on growth, yield and quality of apple trees. 
Optimizing the NPK fertilizer doses is necessary to achieve optimal yield potential. Keeping this in view, the study was undertaken to find out the optimization of quality attributes of High Density Apple cv. Red velox by application of different levels and combinations of $\mathrm{N}, \mathrm{P}$ and $\mathrm{K}$ under temperate conditions of Kashmir Valley.

\section{Materials and Methods}

The experiment was carried out at High Density Apple Block Division of Fruit Science, Sher-e-Kashmir University of Agricultural Sciences and Technology of Kashmir, Shalimar, Srinagar during two consecutive years 2017-18 and 2018-19. The experiment was laid out in a factorial randomized block design with three replications and 64 treatment combinations containing four levels of nitrogen $(0,105,140,175 \mathrm{~g} /$ tree $)$ four levels of phosphorous $(0,43,57,71 \mathrm{~g} /$ tree $)$ and four levels of potassium $(0,187,250,312 \mathrm{~g} /$ tree $)$ during the first year and 0 , 146, 194, $243 \mathrm{~g} \mathrm{~N} /$ tree, $0,60,80,100 \mathrm{gP} /$ tree and 0,263,350,438 $\mathrm{g} \mathrm{K} /$ tree during the second year of experimentation. First half of the nitrogen was applied on 21 days before expected bloom and second half after fruit set while as full dose of phosphorus and half dose of potassium was applied 21 days before expected bloom and another half dose of potassium after fruit set. The results were obtained from ten randomly selected fruits from each observational plant at edible ripe stage. Soluble solid content $\left({ }^{0}\right.$ Brix) was determined by using Zeis hand refractometer Reducing sugar and total sugars were determined by "Dubois method" (Sadasivam and Manickam, 1996) ${ }^{[10]}$. The non-reducing sugars were calculated by subtracting values of reducing sugars from values of total sugars and multiplying by factor 0.95 . The data analysis was carried out using the OPSTAT software (1998).

Table 1: Treatment combination details

\begin{tabular}{|c|c|}
\hline $\mathrm{T}_{1}: \mathrm{N}_{0} \mathrm{P}_{0} \mathrm{~K}_{0}$ (Control) & $\mathrm{T}_{17}: \mathrm{N}_{1} \mathrm{P}_{0} \mathrm{~K}_{0}$ \\
\hline $\mathrm{T}_{2}: \mathrm{N}_{0} \mathrm{P}_{0} \mathrm{~K}_{1}$ & $\mathrm{~T}_{18}: \mathrm{N}_{1} \mathrm{P}_{0} \mathrm{~K}_{1}$ \\
\hline $\mathrm{T}_{3}: \mathrm{N}_{0} \mathrm{P}_{0} \mathrm{~K}_{2}$ & $\mathrm{~T}_{19}: \mathrm{N}_{1} \mathrm{P}_{0} \mathrm{~K}_{2}$ \\
\hline $\mathrm{T}_{4}: \mathrm{N}_{0} \mathrm{P}_{0} \mathrm{~K}_{3}$ & $\mathrm{~T}_{20}: \mathrm{N}_{1} \mathrm{P}_{0} \mathrm{~K}_{3}$ \\
\hline $\mathrm{T}_{5}: \mathrm{N}_{0} \mathrm{P}_{1} \mathrm{~K}_{0}$ & $\mathrm{~T}_{21}: \mathrm{N}_{1} \mathrm{P}_{1} \mathrm{~K}_{0}$ \\
\hline $\mathrm{T}_{6}: \mathrm{N}_{0} \mathrm{P}_{1} \mathrm{~K}_{1}$ & $\mathrm{~T}_{22}: \mathrm{N}_{1} \mathrm{P}_{1} \mathrm{~K}_{1}$ \\
\hline $\mathrm{T}_{7}: \mathrm{N}_{0} \mathrm{P}_{1} \mathrm{~K}_{2}$ & $\mathrm{~T}_{23}: \mathrm{N}_{1} \mathrm{P}_{1} \mathrm{~K}_{2}$ \\
\hline $\mathrm{T}_{8}: \mathrm{N}_{0} \mathrm{P}_{1} \mathrm{~K}_{3}$ & $\mathrm{~T}_{24}: \mathrm{N}_{1} \mathrm{P}_{1} \mathrm{~K}_{3}$ \\
\hline $\mathrm{T}_{9}: \mathrm{N}_{0} \mathrm{P}_{2} \mathrm{~K}_{0}$ & $\mathrm{~T}_{25}: \mathrm{N}_{1} \mathrm{P}_{2} \mathrm{~K}_{0}$ \\
\hline $\mathrm{T}_{10}: \mathrm{N}_{0} \mathrm{P}_{2} \mathrm{~K}_{1}$ & $\mathrm{~T}_{26}: \mathrm{N}_{1} \mathrm{P}_{2} \mathrm{~K}_{1}$ \\
\hline $\mathrm{T}_{11}: \mathrm{N}_{0} \mathrm{P}_{2} \mathrm{~K}_{2}$ & $\mathrm{~T}_{27}: \mathrm{N}_{1} \mathrm{P}_{2} \mathrm{~K}_{2}$ \\
\hline $\mathrm{T}_{12}: \mathrm{N}_{0} \mathrm{P}_{2} \mathrm{~K}_{3}$ & $\mathrm{~T}_{28}: \mathrm{N}_{1} \mathrm{P}_{2} \mathrm{~K}_{3}$ \\
\hline $\mathrm{T}_{13}: \mathrm{N}_{0} \mathrm{P}_{3} \mathrm{~K}_{0}$ & $\mathrm{~T}_{29}: \mathrm{N}_{1} \mathrm{P}_{3} \mathrm{~K}_{0}$ \\
\hline $\mathrm{T}_{14}: 4 \mathrm{dxN}_{0} \mathrm{P}_{3} \mathrm{~K}_{1}$ & $\mathrm{~T}_{30}: \mathrm{N}_{1} \mathrm{P}_{3} \mathrm{~K}_{1}$ \\
\hline $\mathrm{T}_{15}: \mathrm{N}_{0} \mathrm{P}_{3} \mathrm{~K}_{2}$ & $\mathrm{~T}_{31}: \mathrm{N}_{1} \mathrm{P}_{3} \mathrm{~K}_{2}$ \\
\hline $\mathrm{T}_{16}: \mathrm{N}_{0} \mathrm{P}_{3} \mathrm{~K}_{3}$ & $\mathrm{~T}_{32}: \mathrm{N}_{1} \mathrm{P}_{3} \mathrm{~K}_{3}$ \\
\hline $\mathrm{T}_{33}: \mathrm{N}_{2} \mathrm{P}_{0} \mathrm{~K}_{0}$ & $\mathrm{~T}_{49}: \mathrm{N}_{3} \mathrm{P}_{0} \mathrm{~K}_{0}$ \\
\hline $\mathrm{T}_{34}: \mathrm{N}_{2} \mathrm{P}_{0} \mathrm{~K}_{1}$ & $\mathrm{~T}_{50}: \mathrm{N}_{3} \mathrm{P}_{0} \mathrm{~K}_{1}$ \\
\hline $\mathrm{T}_{35}: \mathrm{N}_{2} \mathrm{P}_{0} \mathrm{~K}_{2}$ & $\mathrm{~T}_{51}: \mathrm{N}_{3} \mathrm{P}_{0} \mathrm{~K}_{2}$ \\
\hline $\mathrm{T}_{36}: \mathrm{N}_{2} \mathrm{P}_{0} \mathrm{~K}_{3}$ & $\mathrm{~T}_{52}: \mathrm{N}_{3} \mathrm{P}_{0} \mathrm{~K}_{3}$ \\
\hline $\mathrm{T}_{37}: \mathrm{N}_{2} \mathrm{P}_{1} \mathrm{~K}_{0}$ & $\mathrm{~T}_{53}: \mathrm{N}_{3} \mathrm{P}_{1} \mathrm{~K}_{0}$ \\
\hline $\mathrm{T}_{38}: \mathrm{N}_{2} \mathrm{P}_{1} \mathrm{~K}_{1}$ & $\mathrm{~T}_{54}: \mathrm{N}_{3} \mathrm{P}_{1} \mathrm{~K}_{1}$ \\
\hline $\mathrm{T}_{39}: \mathrm{N}_{2} \mathrm{P}_{1} \mathrm{~K}_{2}$ & $\mathrm{~T}_{55}: \mathrm{N}_{3} \mathrm{P}_{1} \mathrm{~K}_{2}$ \\
\hline $\mathrm{T}_{40}: \mathrm{N}_{2} \mathrm{P}_{1} \mathrm{~K}_{3}$ & $\mathrm{~T}_{56}: \mathrm{N}_{3} \mathrm{P}_{1} \mathrm{~K}_{3}$ \\
\hline $\mathrm{T}_{41}: \mathrm{N}_{2} \mathrm{P}_{2} \mathrm{~K}_{0}$ & $\mathrm{~T}_{57}: \mathrm{N}_{3} \mathrm{P}_{2} \mathrm{~K}_{0}$ \\
\hline $\mathrm{T}_{42}: \mathrm{N}_{2} \mathrm{P}_{2} \mathrm{~K}_{1}$ & $\mathrm{~T}_{58}: \mathrm{N}_{3} \mathrm{P}_{2} \mathrm{~K}_{1}$ \\
\hline $\mathrm{T}_{43}: \mathrm{N}_{2} \mathrm{P}_{2} \mathrm{~K}_{2}$ & $\mathrm{~T}_{59}: \mathrm{N}_{3} \mathrm{P}_{2} \mathrm{~K}_{2}$ \\
\hline $\mathrm{T}_{44}: \mathrm{N}_{2} \mathrm{P}_{2} \mathrm{~K}_{3}$ & $\mathrm{~T}_{60}: \mathrm{N}_{3} \mathrm{P}_{2} \mathrm{~K}_{3}$ \\
\hline $\mathrm{T}_{45}: \mathrm{N}_{2} \mathrm{P}_{3} \mathrm{~K}_{0}$ & $\mathrm{~T}_{61}: \mathrm{N}_{3} \mathrm{P}_{3} \mathrm{~K}_{0}$ \\
\hline $\mathrm{T}_{46}: \mathrm{N}_{2} \mathrm{P}_{3} \mathrm{~K}_{1}$ & $\mathrm{~T}_{62}: \mathrm{N}_{3} \mathrm{P}_{3} \mathrm{~K}_{1}$ \\
\hline $\mathrm{T}_{47}: \mathrm{N}_{2} \mathrm{P}_{3} \mathrm{~K}_{2}$ & $\mathrm{~T}_{63}: \mathrm{N}_{3} \mathrm{P}_{3} \mathrm{~K}_{2}$ \\
\hline $\mathrm{T}_{48}: \mathrm{N}_{2} \mathrm{P}_{3} \mathrm{~K}_{3}$ & $\mathrm{~T}_{64}: \mathrm{N}_{3} \mathrm{P}_{3} \mathrm{~K}_{3}$ \\
\hline
\end{tabular}

\section{Results and Discussion}

In the present investigation combination of different levels of nitrogen, phosphorus and potassium showed improvement in fruit quality of apple Perusal of data presented in Table 2, 3, 4 and 5 revealed that the quality attributes of apple were significantly influenced by the increasing levels of applied nutrients. Among the all treatment combinations, $\mathrm{N}_{2} \mathrm{P}_{3} \mathrm{~K}_{3}$ recorded maximum SSC (14.67and $15.50{ }^{0} \mathrm{Brix}$ ) which was significantly higher than other $\mathrm{N} \times \mathrm{P} \times \mathrm{K}$ treatment combinations followed by $\mathrm{N}_{2} \mathrm{P}_{3} \mathrm{~K}_{2}$ (14.61 and $\left.15.46^{\circ} \mathrm{Brix}\right)$ and the minimum value (13.40 and $\left.14.05{ }^{\circ} \mathrm{Brix}\right)$ was recorded in $\mathrm{N}_{0} \mathrm{P}_{0} \mathrm{~K}_{0}$ during 2017 and 2018 respectively. Maximum total sugars $(9.810$ and $10.05 \%)$ and reducing sugar (7.200 and $7.395 \%$ ) were recorded with combination treatment $\mathrm{N}_{2} \mathrm{P}_{3} \mathrm{~K}_{3}$ (followed by $\mathrm{N}_{2} \mathrm{P}_{3} \mathrm{~K}_{2}$ minimum total sugars (9.170 and $9.300 \%)$ and reducing sugars (6.740 and 6.857\%) were recorded with $\mathrm{N}_{3} \mathrm{P}_{0} \mathrm{~K}_{0}$ during both of the experimental years. However,maximum non-reducing sugars was recorded with combination treatment $\mathrm{N}_{2} \mathrm{P}_{3} \mathrm{~K}_{3}(2.615$ and $2.900 \%)$ followed by $\mathrm{N}_{2} \mathrm{P}_{3} \mathrm{~K}_{2}(2.590$ and $2.875 \%)$ whereas minimum results was recorded with $\mathrm{N}_{3} \mathrm{P}_{0} \mathrm{~K}_{0}(2.122$ and $2.330 \%)$. Quality parameters in terms of SSC, total sugars, reducing and non-reducing sugars seems to be improved by application of different nutrient combinations. High rates of nitrogen fertilizer decreased soluble solids (Nava et al., 2008) [7]. Increasing rates of phosphorus and potassium increase the concentration of soluble solids in the apple flesh (Nava et al., 2008) ${ }^{[7]}$. This might be due to the fact that optimum nitrogen increases the availability of assimilates and higher dose cause excessive vegetative growth which requires most of the metabolites while little was left for storage in the fruit. Nitrogen stimulates the functioning of number of enzymes in the physiological processes, which might have improved the total soluble solid content of the fruits. The finding in line with those of El- Gazzar (2000), Nava et al. (2008) ${ }^{[3,7]}$ and Imam and Brifkany (2010) ${ }^{[5]}$. The highest improvement in total soluble solids was observed in higher levels of phosphorus and potassium. It may be due to the role of phosphorus and potassium in enhancing rate of hydrolysis of polysaccharides into mono-saccharides. This increase in SSS is also due to role of potassium in translocation of sugars into the fruit (Sobulo and Olorunda, 1977) ${ }^{[12]}$. These findings are also in confirmation with Singh et al., (2006) ${ }^{[14]}$ who recorded highest juice percentage, TSS, and highest reducing sugar with $125 \%$ of recommended dose in pomegranate. The effect of nitrogen level $\mathrm{N}_{2}$ on total sugars was found to be most significant due to maximum photosynthates produced in this treatment (Stamper et al. (2003), Hudina et al. (2002) ${ }^{[4],}$ Naiema (2003) ${ }^{[8]}$ and Sharma et al. (2014) ${ }^{[11]}$. The useful effect of phosphorus on quality parameters like sugars was due to the fact that it increased the efficiency of metabolic and physiological processes of plants and thus improved the chemical quality of apple fruits. Highest percentages of total sugars were registered in potassium level $\mathrm{K}_{3}$. This may be attributed to the increased rate of hydrolysis of polysaccharides to monosaccharides by potassium fertilization. Potassium is widely regarded as element required to improve quality of fruits and in this study it is established that the application of potassium is necessary in fruit orchard to harvest good quality crop. Similar results were reported by Stamper et al. (2002) ${ }^{[4]}$ in apple. Singh et al. (2009) also found phosphorus along with nitrogen at lower level coupled with high level of potassium to directly influence all the physico- chemical fruit characters in apple cv. Red delicious. 
Table 2: Influence of different levels and combinations of N, P and K on soluble solids content ( $\left.{ }^{\circ} \mathrm{brix}\right)$ of apple cv. Red velox.

\begin{tabular}{|c|c|c|c|c|c|c|c|c|c|c|c|}
\hline \multirow{3}{*}{ Nitrogen } & \multirow{3}{*}{ Phosphorus } & \multicolumn{10}{|c|}{ Potassium } \\
\hline & & & & 2017-20 & & & & & $018-20$ & & \\
\hline & & $\mathbf{K}_{\mathbf{0}}$ & $\mathbf{K}_{1}$ & $\mathbf{K}_{2}$ & $\mathbf{K}_{3}$ & Mean & $\mathbf{K}_{\mathbf{0}}$ & $K_{1}$ & $\mathbf{K}_{2}$ & $\mathbf{K}_{3}$ & Mean \\
\hline \multirow{5}{*}{$\mathrm{N}_{0}$} & $\mathrm{P}_{0}$ & 13.40 & 13.60 & 13.80 & 13.90 & 13.67 & 14.05 & 14.12 & 14.18 & 14.22 & 14.14 \\
\hline & $\mathrm{P}_{1}$ & 13.60 & 13.80 & 13.95 & 14.00 & 13.83 & 14.15 & 14.18 & 14.23 & 14.26 & 14.20 \\
\hline & $\mathrm{P}_{2}$ & 13.85 & 14.00 & 14.10 & 14.15 & 14.02 & 14.20 & 14.24 & 14.28 & 14.33 & 14.26 \\
\hline & $\mathrm{P}_{3}$ & 14.00 & 14.10 & 14.15 & 14.20 & 14.11 & 14.24 & 14.30 & 14.35 & 14.40 & 14.32 \\
\hline & Sub Means & 13.71 & 13.87 & 14.00 & 14.06 & 13.91 & 14.16 & 14.21 & 14.26 & 14.30 & 14.23 \\
\hline \multirow{5}{*}{$\mathrm{N}_{1}$} & $\mathrm{P}_{0}$ & 14.03 & 14.05 & 14.08 & 14.10 & 14.06 & 14.65 & 14.67 & 14.69 & 14.70 & 14.67 \\
\hline & $\mathrm{P}_{1}$ & 14.06 & 14.09 & 14.11 & 14.16 & 14.10 & 14.68 & 14.70 & 14.73 & 14.78 & 14.72 \\
\hline & $\mathrm{P}_{2}$ & 14.10 & 14.12 & 14.17 & 14.20 & 14.14 & 14.70 & 14.75 & 14.85 & 14.87 & 14.79 \\
\hline & $\mathrm{P}_{3}$ & 14.15 & 14.18 & 14.22 & 14.25 & 14.20 & 14.80 & 14.87 & 14.90 & 14.97 & 14.88 \\
\hline & Sub Means & 14.08 & 14.11 & 14.14 & 14.17 & 14.12 & 14.70 & 14.74 & 14.79 & 14.83 & 14.76 \\
\hline \multirow{5}{*}{$\mathrm{N}_{2}$} & $\mathrm{P}_{0}$ & 14.20 & 14.23 & 14.25 & 14.28 & 14.24 & 14.85 & 14.90 & 14.94 & 14.97 & 14.91 \\
\hline & $\mathrm{P}_{1}$ & 14.24 & 14.25 & 14.30 & 14.32 & 14.27 & 14.92 & 14.95 & 14.99 & 15.03 & 14.97 \\
\hline & $\mathrm{P}_{2}$ & 14.27 & 14.31 & 14.35 & 14.38 & 14.32 & 14.98 & 15.05 & 15.10 & 15.17 & 15.07 \\
\hline & $\mathrm{P}_{3}$ & 14.32 & 14.36 & 14.42 & 14.50 & 14.40 & 15.10 & 15.20 & 15.25 & 15.29 & 15.21 \\
\hline & Sub Means & 14.25 & 14.28 & 14.33 & 14.37 & 14.31 & 14.96 & 15.02 & 15.07 & 15.11 & 15.04 \\
\hline \multirow{7}{*}{$\mathrm{N}_{3}$} & $\mathrm{P}_{0}$ & 14.10 & 14.15 & 14.20 & 14.25 & 14.17 & 14.75 & 14.80 & 14.85 & 14.91 & 14.82 \\
\hline & $\mathrm{P}_{1}$ & 14.18 & 14.23 & 14.26 & 14.28 & 14.23 & 14.82 & 14.87 & 14.91 & 14.97 & 14.89 \\
\hline & $\mathrm{P}_{2}$ & 14.25 & 14.30 & 14.31 & 14.35 & 14.30 & 14.90 & 14.95 & 15.00 & 15.08 & 14.98 \\
\hline & $\mathrm{P}_{3}$ & 14.30 & 14.34 & 14.37 & 14.44 & 14.36 & 15.01 & 15.10 & 15.15 & 15.21 & 15.10 \\
\hline & Sub Means & 14.20 & 14.25 & 14.28 & 14.33 & 14.27 & 14.87 & 14.93 & 14.97 & 15.04 & 14.95 \\
\hline & Potassium & 14.06 & 14.12 & 14.18 & 14.23 & & 14.67 & 14.72 & 14.77 & 14.82 & \\
\hline & Phosphorus & 14.03 & 14.10 & 14.19 & 14.26 & & 14.66 & 14.69 & 14.77 & 14.87 & \\
\hline & & \multicolumn{5}{|c|}{$\begin{array}{c}\text { CD }(p \leq 0.05) \\
\text { Nitrogen (N): } 0.07 \\
\text { Phosphorus(P): } 0.07 \\
\text { Potassium (K): } 0.07 \\
\text { N×P: N.S } \\
\text { N×K: N.S } \\
\text { K×P: } 0.14 \\
\text { N×P×K: } 0.29 \\
\end{array}$} & \multicolumn{5}{|c|}{$\begin{array}{c}\mathrm{CD}(p \leq 0.05) \\
\text { Nitrogen }(\mathrm{N}): 0.01 \\
\text { Phosphorus(P): } 0.01 \\
\text { Potassium }(\mathrm{K}): 0.01 \\
\mathrm{~N} \times \mathrm{P}: \mathrm{N} . \mathrm{S} \\
\mathrm{N} \times \mathrm{K}: \mathrm{N} . \mathrm{S} \\
\mathrm{K} \times \mathrm{P}: 0.02 \\
\mathrm{~N} \times \mathrm{P} \times \mathrm{K}: 0.05 \\
\end{array}$} \\
\hline
\end{tabular}

Table 3: Influence of different levels and combinations of N, P and K on total sugars (\%) of apple cv. Red velox.

\begin{tabular}{|c|c|c|c|c|c|c|c|c|c|c|c|}
\hline \multirow{3}{*}{ Nitrogen } & \multirow{3}{*}{ Phosphorus } & \multicolumn{10}{|c|}{ Potassium } \\
\hline & & \multicolumn{5}{|c|}{ 2017-2018 } & \multicolumn{5}{|c|}{ 2018-2019 } \\
\hline & & $\mathbf{K}_{\mathbf{0}}$ & $\mathbf{K}_{1}$ & $\mathbf{K}_{2}$ & $\overline{K_{3}}$ & Mean & $\mathbf{K}_{\mathbf{0}}$ & $\overline{K_{1}}$ & $\mathbf{K}_{2}$ & $\mathbf{K}_{\mathbf{3}}$ & Mean \\
\hline \multirow{5}{*}{$\mathrm{N}_{0}$} & $\mathrm{P}_{0}$ & 9.170 & 9.200 & 9.260 & 9.300 & 9.232 & 9.300 & 9.350 & 9.380 & 9.450 & 9.370 \\
\hline & $\mathrm{P}_{1}$ & 9.220 & 9.270 & 9.320 & 9.370 & 9.295 & 9.360 & 9.400 & 9.470 & 9.550 & 9.445 \\
\hline & $\mathrm{P}_{2}$ & 9.290 & 9.330 & 9.400 & 9.450 & 9.367 & 9.420 & 9.500 & 9.580 & 9.640 & 9.535 \\
\hline & $\mathrm{P}_{3}$ & 9.350 & 9.410 & 9.470 & 9.550 & 9.445 & 9.530 & 9.600 & 9.690 & 9.750 & 9.642 \\
\hline & Sub Means & 9.257 & 9.302 & 9.362 & 9.417 & 9.335 & 9.402 & 9.462 & 9.530 & 9.597 & 9.498 \\
\hline \multirow{5}{*}{$\mathrm{N}_{1}$} & $\mathrm{P}_{0}$ & 9.200 & 9.240 & 9.300 & 9.340 & 9.270 & 9.400 & 9.460 & 9.500 & 9.580 & 9.485 \\
\hline & $\mathrm{P}_{1}$ & 9.260 & 9.300 & 9.360 & 9.390 & 9.327 & 9.480 & 9.520 & 9.600 & 9.650 & 9.562 \\
\hline & $\mathrm{P}_{2}$ & 9.320 & 9.360 & 9.420 & 9.500 & 9.400 & 9.540 & 9.600 & 9.670 & 9.760 & 9.642 \\
\hline & $\mathrm{P}_{3}$ & 9.400 & 9.445 & 9.520 & 9.580 & 9.486 & 9.650 & 9.750 & 9.800 & 9.860 & 9.765 \\
\hline & Sub Means & 9.295 & 9.336 & 9.400 & 9.452 & 9.370 & 9.5175 & 9.582 & 9.642 & 9.712 & 9.613 \\
\hline \multirow{5}{*}{$\mathrm{N}_{2}$} & $\mathrm{P}_{0}$ & 9.330 & 9.400 & 9.470 & 9.510 & 9.427 & 9.580 & 9.620 & 9.670 & 9.720 & 9.647 \\
\hline & $\mathrm{P}_{1}$ & 9.420 & 9.470 & 9.530 & 9.600 & 9.505 & 9.640 & 9.700 & 9.740 & 9.790 & 9.717 \\
\hline & $\mathrm{P}_{2}$ & 9.490 & 9.550 & 9.630 & 9.710 & 9.595 & 9.700 & 9.770 & 9.850 & 9.900 & 9.805 \\
\hline & $\mathrm{P}_{3}$ & 9.560 & 9.650 & 9.720 & 9.810 & 9.685 & 9.770 & 9.850 & 9.920 & 10.05 & 9.897 \\
\hline & Sub Means & 9.450 & 9.517 & 9.587 & 9.657 & 9.553 & 9.672 & 9.735 & 9.795 & 9.865 & 9.766 \\
\hline \multirow{7}{*}{$\mathrm{N}_{3}$} & $\mathrm{P}_{0}$ & 9.040 & 9.080 & 9.110 & 9.160 & 9.097 & 9.110 & 9.160 & 9.210 & 9.270 & 9.187 \\
\hline & $\mathrm{P}_{1}$ & 9.090 & 9.140 & 9.190 & 9.220 & 9.160 & 9.170 & 9.230 & 9.290 & 9.360 & 9.262 \\
\hline & $\mathrm{P}_{2}$ & 9.150 & 9.190 & 9.240 & 9.280 & 9.215 & 9.250 & 9.300 & 9.380 & 9.450 & 9.345 \\
\hline & $\mathrm{P}_{3}$ & 9.200 & 9.250 & 9.300 & 9.350 & 9.275 & 9.320 & 9.400 & 9.500 & 9.570 & 9.447 \\
\hline & Sub Means & 9.120 & 9.165 & 9.210 & 9.252 & 9.186 & 9.212 & 9.272 & 9.345 & 9.412 & 9.310 \\
\hline & Potassium & 9.256 & 9.321 & 9.394 & 9.472 & & 9.422 & 9.496 & 9.581 & 9.687 & \\
\hline & Phosphorus & 9.280 & 9.330 & 9.389 & 9.444 & & 9.450 & 9.512 & 9.578 & 9.646 & \\
\hline & & \multicolumn{5}{|c|}{$\begin{array}{c}\text { CD }(p \leq 0.05) \\
\text { Nitrogen }(\mathrm{N}): 0.16 \\
\text { Phosphorus }(\mathrm{P}): 0.16 \\
\text { Potassium (K): } 0.16 \\
\text { N×P: N.S } \\
\text { N×K: } 0.33 \\
\text { K×P: N.S } \\
\text { N } \times \text { P } \times \text { K: } 0.66\end{array}$} & \multicolumn{5}{|c|}{$\begin{array}{c}\mathrm{CD}(p \leq 0.05) \\
\text { Nitrogen }(\mathrm{N}): 0.03 \\
\text { Phosphorus (P): } 0.03 \\
\text { Potassium (K): } 0.03 \\
\mathrm{~N} \times \mathrm{P}: \mathrm{N} . \mathrm{S} \\
\mathrm{N} \times \mathrm{K}: 0.05 \\
\mathrm{~K} \times \mathrm{P}: \mathrm{N} . \mathrm{S} \\
\mathrm{N} \times \mathrm{P} \times \mathrm{K}: 0.11\end{array}$} \\
\hline
\end{tabular}


Table 4: Influence of different levels and combinations of N, P and K on reducing sugars (\%) of apple cv. Red velox.

\begin{tabular}{|c|c|c|c|c|c|c|c|c|c|c|c|}
\hline \multirow{3}{*}{ Nitrogen } & \multirow{3}{*}{ Phosphorus } & \multicolumn{10}{|c|}{ Potassium } \\
\hline & & & & 2017-20 & & & & & 2018-20 & & \\
\hline & & $\mathbf{K}_{\mathbf{0}}$ & $\mathbf{K}_{1}$ & $\mathbf{K}_{2}$ & $\mathrm{~K}_{3}$ & Mean & $\mathbf{K}_{\mathbf{0}}$ & $\mathbf{K}_{1}$ & $\mathbf{K}_{2}$ & $\mathrm{~K}_{3}$ & Mean \\
\hline \multirow{5}{*}{$\mathrm{N}_{0}$} & $\mathrm{P}_{0}$ & 6.780 & 6.805 & 6.840 & 6.880 & 6.826 & 6.928 & 6.948 & 6.965 & 7.003 & 6.961 \\
\hline & $\mathrm{P}_{1}$ & 6.805 & 6.855 & 6.890 & 6.930 & 6.870 & 6.955 & 6.975 & 7.010 & 7.035 & 6.993 \\
\hline & $\mathrm{P}_{2}$ & 6.870 & 6.900 & 6.955 & 6.990 & 6.928 & 7.000 & 7.016 & 7.050 & 7.007 & 7.018 \\
\hline & $\mathrm{P}_{3}$ & 6.910 & 6.965 & 7.000 & 7.050 & 6.981 & 7.032 & 7.065 & 7.090 & 7.127 & 7.078 \\
\hline & Sub Means & 6.841 & 6.881 & 6.921 & 6.962 & 6.901 & 6.978 & 7.001 & 7.028 & 7.043 & 7.012 \\
\hline \multirow{5}{*}{$\mathrm{N}_{1}$} & $\mathrm{P}_{0}$ & 6.800 & 6.840 & 6.865 & 6.920 & 6.856 & 6.810 & 6.830 & 6.880 & 6.930 & 6.862 \\
\hline & $\mathrm{P}_{1}$ & 6.853 & 6.885 & 6.915 & 6.970 & 6.905 & 6.830 & 6.875 & 6.915 & 6.930 & 6.887 \\
\hline & $\mathrm{P}_{2}$ & 6.890 & 6.920 & 6.985 & 7.055 & 6.962 & 6.890 & 6.910 & 6.845 & 7.010 & 6.913 \\
\hline & $\mathrm{P}_{3}$ & 6.960 & 7.000 & 7.060 & 7.090 & 7.027 & 6.930 & 6.960 & 7.010 & 7.050 & 6.987 \\
\hline & Sub Means & 6.875 & 6.911 & 6.956 & 7.008 & 6.938 & 6.865 & 6.893 & 6.912 & 6.980 & 6.912 \\
\hline \multirow{5}{*}{$\mathrm{N}_{2}$} & $\mathrm{P}_{0}$ & 6.830 & 6.855 & 6.870 & 6.900 & 6.863 & 6.830 & 6.887 & 6.945 & 6.970 & 6.908 \\
\hline & $\mathrm{P}_{1}$ & 6.870 & 6.900 & 6.920 & 6.955 & 6.911 & 6.903 & 6.945 & 6.980 & 7.035 & 6.965 \\
\hline & $\mathrm{P}_{2}$ & 6.900 & 6.915 & 6.950 & 7.010 & 6.943 & 6.957 & 7.008 & 7.055 & 7.125 & 7.036 \\
\hline & $\mathrm{P}_{3}$ & 6.940 & 6.960 & 7.025 & 7.200 & 7.031 & 7.010 & 7.075 & 7.130 & 7.395 & 7.152 \\
\hline & Sub Means & 6.885 & 6.907 & 6.941 & 7.016 & 6.937 & 6.925 & 6.978 & 7.027 & 7.131 & 7.015 \\
\hline \multirow{7}{*}{$\mathrm{N}_{3}$} & $\mathrm{P}_{0}$ & 6.740 & 6.760 & 6.800 & 6.910 & 6.802 & 6.857 & 6.868 & 6.922 & 6.949 & 6.899 \\
\hline & $\mathrm{P}_{1}$ & 6.780 & 6.800 & 6.910 & 6.950 & 6.860 & 6.898 & 6.930 & 6.964 & 6.995 & 6.946 \\
\hline & $\mathrm{P}_{2}$ & 6.850 & 6.930 & 6.960 & 6.970 & 6.927 & 6.948 & 6.971 & 7.015 & 7.065 & 6.999 \\
\hline & $\mathrm{P}_{3}$ & 6.965 & 7.000 & 6.980 & 6.990 & 6.983 & 6.989 & 7.030 & 7.058 & 7.138 & 7.053 \\
\hline & Sub Means & 6.833 & 6.872 & 6.912 & 6.955 & 6.893 & 6.923 & 6.949 & 6.989 & 7.036 & 6.974 \\
\hline & Potassium & 6.836 & 6.886 & 6.940 & 7.005 & & 6.907 & 6.947 & 6.991 & 7.067 & \\
\hline & Phosphorus & 6.858 & 6.892 & 6.932 & 6.985 & & 6.922 & 6.955 & 6.989 & 7.047 & \\
\hline & & \multicolumn{5}{|c|}{$\begin{array}{c}\mathrm{CD}(p \leq 0.05) \\
\text { Nitrogen }(\mathrm{N}): 0.14 \\
\text { Phosphorus }(\mathrm{P}): 0.14 \\
\text { Potassium }(\mathrm{K}): \mathrm{N} . \mathrm{S} \\
\mathrm{N} \times \mathrm{P}: 0.30 \\
\mathrm{~N} \times \mathrm{K}: \mathrm{N} . \mathrm{S} \\
\mathrm{K} \times \mathrm{P}: \mathrm{N} . \mathrm{S} \\
\mathrm{N} \times \mathrm{P} \times \mathrm{K}: 0.60\end{array}$} & \multicolumn{5}{|c|}{$\begin{array}{c}\text { Nitrogen }(\mathrm{N}): 0.08 \\
\text { Phosphorus (P): } 0.08 \\
\text { Potassium (K): N.S } \\
\text { N×P: } 0.17 \\
\text { N×K: N.S } \\
\text { K×P: N.S } \\
\text { N×P×K: } 0.35\end{array}$} \\
\hline
\end{tabular}

Table 5: Influence of different levels and combinations of N, P and K on non-reducing sugars (\%) of apple cv. Red velox.

\begin{tabular}{|c|c|c|c|c|c|c|c|c|c|c|c|}
\hline \multirow{3}{*}{ Nitrogen } & \multirow{3}{*}{ Phosphorus } & \multicolumn{10}{|c|}{ Potassium } \\
\hline & & & & 2017-20 & & & & & 2018-20 & & \\
\hline & & $\mathbf{K}_{\mathbf{0}}$ & $\mathbf{K}_{1}$ & $\mathbf{K}_{2}$ & $\mathbf{K}_{3}$ & Mean & $\mathbf{K}_{\mathbf{0}}$ & $\mathbf{K}_{1}$ & $\mathbf{K}_{2}$ & $\mathbf{K}_{3}$ & Mean \\
\hline \multirow{5}{*}{$\mathrm{N}_{0}$} & $\mathrm{P}_{0}$ & 2.270 & 2.275 & 2.299 & 2.299 & 2.285 & 2.348 & 2.376 & 2.390 & 2.403 & 2.379 \\
\hline & $\mathrm{P}_{1}$ & 2.294 & 2.294 & 2.308 & 2.318 & 2.303 & 2.379 & 2.398 & 2.432 & 2.451 & 2.415 \\
\hline & $\mathrm{P}_{2}$ & 2.299 & 2.308 & 2.322 & 2.337 & 2.316 & 2.394 & 2.432 & 2.451 & 2.460 & 2.434 \\
\hline & $\mathrm{P}_{3}$ & 2.318 & 2.322 & 2.346 & 2.375 & 2.340 & 2.403 & 2.441 & 2.470 & 2.480 & 2.448 \\
\hline & Sub Means & 2.295 & 2.300 & 2.318 & 2.332 & 2.311 & 2.381 & 2.411 & 2.435 & 2.448 & 2.419 \\
\hline \multirow{5}{*}{$\mathrm{N}_{1}$} & $\mathrm{P}_{0}$ & 2.280 & 2.280 & 2.313 & 2.318 & 2.297 & 2.441 & 2.460 & 2.470 & 2.498 & 2.467 \\
\hline & $\mathrm{P}_{1}$ & 2.286 & 2.294 & 2.322 & 2.327 & 2.307 & 2.470 & 2.512 & 2.527 & 2.536 & 2.511 \\
\hline & $\mathrm{P}_{2}$ & 2.308 & 2.318 & 2.327 & 2.337 & 2.322 & 2.490 & 2.517 & 2.536 & 2.555 & 2.524 \\
\hline & $\mathrm{P}_{3}$ & 2.318 & 2.322 & 2.346 & 2.365 & 2.337 & 2.527 & 2.565 & 2.565 & 2.575 & 2.558 \\
\hline & Sub Means & 2.298 & 2.303 & 2.327 & 2.336 & 2.316 & 2.482 & 2.513 & 2.524 & 2.541 & 2.515 \\
\hline \multirow{5}{*}{$\mathrm{N}_{2}$} & $\mathrm{P}_{0}$ & 2.375 & 2.417 & 2.470 & 2.479 & 2.435 & 2.584 & 2.596 & 2.617 & 2.622 & 2.604 \\
\hline & $\mathrm{P}_{1}$ & 2.422 & 2.441 & 2.479 & 2.512 & 2.463 & 2.603 & 2.617 & 2.622 & 2.636 & 2.619 \\
\hline & $\mathrm{P}_{2}$ & 2.460 & 2.503 & 2.546 & 2.565 & 2.518 & 2.612 & 2.631 & 2.655 & $2 . .670$ & 2.632 \\
\hline & $\mathrm{P}_{3}$ & 2.489 & 2.555 & 2.560 & 2.612 & 2.554 & 2.622 & 2.637 & 2.670 & 2.736 & 2.666 \\
\hline & Sub Means & 2.436 & 2.479 & 2.513 & 2.542 & 2.492 & 2.605 & 2.620 & 2.641 & 2.664 & 2.632 \\
\hline \multirow{7}{*}{$\mathrm{N}_{3}$} & $\mathrm{P}_{0}$ & 2.185 & 2.204 & 2.218 & 2.232 & 2.209 & 2.140 & 2.177 & 2.194 & 2.204 & 2.178 \\
\hline & $\mathrm{P}_{1}$ & 2.194 & 2.223 & 2.242 & 2.251 & 2.227 & 2.156 & 2.185 & 2.209 & 2.256 & 2.201 \\
\hline & $\mathrm{P}_{2}$ & 2.223 & 2.232 & 2.251 & 2.261 & 2.241 & 2.186 & 2.212 & 2.246 & 2.265 & 2.227 \\
\hline & $\mathrm{P}_{3}$ & 2.232 & 2.251 & 2.261 & 2.280 & 2.256 & 2.214 & 2.251 & 2.319 & 2.327 & 2.277 \\
\hline & Sub Means & 2.208 & 2.227 & 2.243 & 2.256 & 2.233 & 2.174 & 2.206 & 2.242 & 2.263 & 2.221 \\
\hline & Potassium & 2.306 & 2.325 & 2.349 & 2.371 & & 2.407 & 2.436 & 2.466 & 2.473 & \\
\hline & Phosphorus & 2.309 & 2.327 & 2.350 & 2.366 & & 2.410 & 2.437 & 2.460 & 2.479 & \\
\hline & & \multicolumn{5}{|c|}{$\begin{array}{c}\text { CD }(p \leq 0.05) \\
\text { Nitrogen }(\mathrm{N}): 0.03 \\
\text { Phosphorus }(\mathrm{P}): 0.03 \\
\text { Potassium }(\mathrm{K}): 0.03 \\
\mathrm{~N} \times \mathrm{P}: 0.04 \\
\mathrm{~N} \times \mathrm{K}: 0.04 \\
\mathrm{~K} \times \mathrm{P}: 0.04 \\
\mathrm{~N} \times \mathrm{P} \times \mathrm{K}: 0.08\end{array}$} & \multicolumn{5}{|c|}{$\begin{array}{c}\mathrm{CD}(p \leq 0.05) \\
\text { Nitrogen }(\mathrm{N}): 0.02 \\
\text { Phosphorus }(\mathrm{P}): 0.02 \\
\text { Potassium }(\mathrm{K}): 0.02 \\
\mathrm{~N} \times \mathrm{P}: 0.05 \\
\mathrm{~N} \times \mathrm{K}: 0.05 \\
\mathrm{~K} \times \mathrm{P}: 0.05 \\
\mathrm{~N} \times \mathrm{P} \times \mathrm{K}: 0.09\end{array}$} \\
\hline
\end{tabular}




\section{References}

1. Anonymous. Area and Production of Fruit plants, Directorate of Horticulture, Govt. of Jammu \& Kashmir, Srinagar, 2019.

2. AOAC. Official and Tentative Methods of Analysis. Association of Official Agricultural Chemists. 15th edition. Washington, D.C., USA, 1990, 484p.

3. El-Gazzar AAM. Effect of fertilization with nitrogen, potassium and magnesium application on Anna apples. Annals of Agricultural Science Cairo. 2000; 3:11451152.

4. Hudina M, Stamper F, Tagliarini M, Toselli MM, Bertschinger L, Thalheimer M. Effect of phosphorous and potassium foliar fertilization on quality of pears. Proceedings of the International Symposium on foliar nutrition of perennial fruit plants, Meran, Italy, Acta Horticulturae. 2002; 594:487-493. [c.f. CAB Abstract2002: 2004].

5. Imam NMAA, Al-Brifkany MA. Effect of nitrogen fertilization and foliar application of boron on fruit set, vegetative growth and yield of 'Anna' apple cultivar (Malus domestica Borkh.). Mesopotamia Journal of Agriculture. 2010; 38(4):1815-1816.

6. Mishra D. Nutrient removal studies in guava under high density orcharding system. Journal of Agriculture and Crop Science. 2014; 1(2):36-38.

7. Nava G, Dechen AR, Nachtigall GR. Nitrogen and potassium fertilization affect apple fruit quality in Southern Brazil. Communication in Soil Science and Plant Analysis. 2008; 39(1-2):96-107.

8. Naiema MS. Effect of different doses of nitrogen and potassium on leaf mineral content, fruit set, yield and fruit quality of apple grown in calcarious soils. Alexandria Journal of Agricultural Research. 2003; 48(1):85-92.

9. Policarpo MG, Talluto RL, Bianco. Vegetative and productive responses of 'Conference' and 'Williams' pear trees planted at different in row spacings. Sci. Hortic. 2006; 109:322-331.

10. Sadasivam S, Manickam A. Biochemical method, 2nd Edn. New Age International Limited Publication, 1996.

11. Sharma, Kumar V, Tiwari R, Chouhan P. Effect of N, P and their interaction on physico-chemical parameters of Guava (Psidium guajava) cv. L-49 under Malwa Plateau conditions. International Journal of Scientific and Research Publications. 2014; 4(11):1-4.

12. Sobulo MJ, Olorunda AO. The effect of nitrogen, phosphorous and potassium on the canning quality of tomatoes (Lycopersicon esculentum) in Southern Nigeria. Acta Horticulturae. 1977; 53:171-180.

13. Singh T, Sandhu AS, Singh R, Dhillon WS. Vegetative and fruiting behaviour of semi-soft pear strains in relation to nutrient status. Indian Journal of Horticulture. 2005; 62(3):231-234

14. Singh SR, Sharma AK, Sharma MK. Effect of different NPK combinations on fruit yield, quality and leaf nutrient composition of apple (Malus domestica Borkh.) cv. Red Delicious at different locations. Environment and Ecology. 2006; 24(1):71-75.

15. Singh JK, Prasad J, Singh HK. Effect of micronutrients and plant growth regulators on yield and physicochemical characteristics of aonla fruit in cv. Narendra Aonla-10. Indian Journal of Horticulture. 2007; 64(2):216-218
16. Stampar F, Hudina M, Usenik V, Starm K, Verber G, Veberic R. Experience with foliar nutrition in apple orchard. Acta Horticulturae. 2002; 594:547-552.

17. Waller WM. Use of apple analysis. Mineral nutrition of fruit trees. Butterworths, London, 1980, 383-394. 